Article

\title{
A Note on the Topological Group $c_{0}$
}

\author{
Michael Megrelishvili \\ Department of Mathematics, Bar-Ilan University, 52900 Ramat-Gan, Israel; megereli@math.biu.ac.il
}

Received: 28 September 2018; Accepted: 24 October 2018; Published: 29 October 2018

\begin{abstract}
A well-known result of Ferri and Galindo asserts that the topological group $c_{0}$ is not reflexively representable and the algebra $\mathrm{WAP}\left(c_{0}\right)$ of weakly almost periodic functions does not separate points and closed subsets. However, it is unknown if the same remains true for a larger important algebra Tame $\left(c_{0}\right)$ of tame functions. Respectively, it is an open question if $c_{0}$ is representable on a Rosenthal Banach space. In the present work we show that Tame $\left(c_{0}\right)$ is small in a sense that the unit sphere $S$ and $2 S$ cannot be separated by a tame function $f \in \operatorname{Tame}\left(c_{0}\right)$. As an application we show that the Gromov's compactification of $c_{0}$ is not a semigroup compactification. We discuss some questions.
\end{abstract}

Keywords: Gromov's compactification; group representation; matrix coefficient; semigroup compactification; tame function

\section{Introduction}

Recall that for every Hausdorff topological group $G$ the algebra WAP $(G)$ of all weakly almost periodic functions on $G$ determines the universal semitopological semigroup compactification $u_{w}$ : $G \rightarrow G^{w}$ of $G$. This map is a topological embedding for many groups including the locally compact case. For some basic material about $\operatorname{WAP}(G)$ we refer to [1,2].

The question if $u_{w}$ always is a topological embedding (i.e., if $\operatorname{WAP}(G)$ determines the topology of $G$ ) was raised by Ruppert [2]. This question was negatively answered in [1] by showing that the Polish topological group $G:=H_{+}[0,1]$ of orientation preserving homeomorphisms of the closed unit interval has only constant WAP functions and that every continuous representation $h: G \rightarrow I s(V)$ (by linear isometries) on a reflexive Banach space $V$ is trivial. The WAP triviality of $H_{+}[0,1]$ was conjectured by Pestov.

Recall also that for $G:=H_{+}[0,1]$ every Asplund (hence also every WAP) function is constant and every continuous representation $G \rightarrow \operatorname{Iso}(V)$ on an Asplund (hence also reflexive) space $V$ must be trivial [3]. In contrast one may show (see $[4,5]$ ) that $H_{+}[0,1]$ is representable on a (separable) Rosenthal space (a Banach space is Rosenthal if it does not contain a subspace topologically isomorphic to $l_{1}$ ).

We have the inclusions of topological G-algebras

$$
\operatorname{WAP}(G) \subset \operatorname{Asp}(G) \subset \operatorname{Tame}(G) \subset \operatorname{RUC}(G) .
$$

For details about Tame $(G)$ and definition of $\operatorname{Asp}(G)$ see [5-7]. We only remark that $f \in \operatorname{Tame}(G)$ if and only if $f$ is a matrix coefficient of a Rosenthal representation. That is, there exist: a Rosenthal Banach space $V$; a continuous homomorphism $h: G \rightarrow I s(V)$ into the topological group of all linear isometries $V \rightarrow V$ with strong operator topology; two vectors $v \in V ; \psi \in V^{*}$ (the dual of $V$ ) such that $f(g)=\psi(h(g) v)$ for every $g \in G$.

Similarly, it can be characterized $f \in \operatorname{Asp}(G)$ replacing Rosenthal spaces by the larger class of Asplund spaces. A Banach space is Asplund if the dual of every separable subspace is separable. Every reflexive space is Asplund and every Asplund is Rosenthal. A standard example of an Asplund but nonreflexive space is just $c_{0}$. 
Recall that $c_{0}$, as an additive abelian topological group, is not representable on a reflexive Banach space by a well-known result of Ferri and Galindo [8]. In fact, $\operatorname{WAP}\left(c_{0}\right)$ separates the points but not points and closed subsets. The group $c_{0}$ admits an injective continuous homomorphism $h: c_{0} \rightarrow I s(V)$ with some reflexive $V$ but such $h$ cannot be a topological embedding.

Presently it is an open question if every topological group (abelian, or not) $G$ is Rosenthal representable and if Tame $(G)$ determines the topology of $G$. Note that the algebra Tame $(G)$ appears as an important modern tool in some new research lines in topological dynamics motivating its detailed study $[5,7]$.

One of the good reasons to study Tame $(G)$ is a special role of tameness in the dynamical Berglund-Fremlin-Talagrand dichotomy [5]; as well as direct links to Rosenthal's $l_{1}$-dychotomy. In a sense Tame $(G)$ is a set of all functions which are not dynamically massive.

By these reasons and since $H_{+}[0,1]$ is Rosenthal representable, it seems to be an attractive concrete question if $c_{0}$ is Rosenthal representable and it is worth studying how large is Tame $\left(c_{0}\right)$. In the present work we show that Tame $\left(c_{0}\right)$ is quite small (even for the discrete copy of $c_{0}$, see Theorem 3 ).

Theorem 1. Tame $\left(c_{0}\right)$ does not separate the unit sphere $S$ and $2 S$.

So, the closures of $S$ and $2 S$ intersect in the universal tame compactification of $c_{0}$ (a fortiori, the same is true for the universal Asplund (HNS) semigroup compactification).

Another interesting question is if $c_{0}$ admits an embedding into a metrizable semigroup compactification. Note that any metrizable semigroup compactification of $H_{+}[0,1]$ is trivial.

In Section 3 we show that the Gromov's compactification $\gamma: c_{0} \hookrightarrow P$, which is metrizable (and $\gamma$ is a $G$-embedding), is not a semigroup compactification.

Theorem 2. Let $\gamma: c_{0} \hookrightarrow P$ be the Gromov's compactification of the metric space $\left(c_{0}, \frac{d}{1+d}\right)$, where $d(x, y):=$ $\|x-y\|$. Then $\gamma$ is not a semigroup compactification.

This gives an example of a naturally defined separable unital (original topology determining) $G$-subalgebra of $\operatorname{RUC}(G)$ (for $G=c_{0}$ ) which is not left m-introverted in the sense of [9].

\section{Tame Functions on $c_{0}$}

Recall that a sequence $f_{n}$ of real-valued functions on a set $X$ is said to be independent if there exist real numbers $a<b$ such that

$$
\bigcap_{n \in P} f_{n}^{-1}(-\infty, a) \cap \bigcap_{n \in M} f_{n}^{-1}(b, \infty) \neq \varnothing
$$

for all finite disjoint subsets $P, M$ of $\mathbb{N}$. Every bounded independent sequence is an $l_{1}$-sequence [10].

As in $[6,7]$ we say that a bounded family $F$ of real-valued (not necessarily continuous) functions on a set $X$ is a tame family if $F$ does not contain an independent sequence.

Let $G$ be a topological group, $f: G \rightarrow \mathbb{R}$ be a real-valued function. For every $g \in G$ define $f g: G \rightarrow \mathbb{R}$ as $(f g)(x)=f(g x)$ (for multiplicative $G$ ). Denote by RUC $(G)$ the algebra of all bounded right uniformly continuous functions on $G$. So, $f \in \operatorname{RUC}(G)$ means that $f$ is bounded and for every $\epsilon>0$ there exists a neighborhood $U$ of the identity $e$ (of the multiplicative group $G$ ) such that $|f(u x)-f(x)|<\epsilon$ for every $x \in G$ and $u \in U$. This algebra RUC $(G)$ corresponds to the greatest $G$-compactification $G \rightarrow \beta_{G} G$ of $G$ (with respect to the left action), greatest ambit of $G$.

We say that $f \in \operatorname{RUC}(G)$ is a tame function if the orbit $f G:=\{f g\}_{g \in G}$ is a tame family. That is, $f G$ does not contain an independent sequence; notation $f \in \operatorname{Tame}(G)$. 


\subsection{Proof of Theorem 1}

We have to show that Tame $\left(c_{0}\right)$ does not separate the spheres $S$ and $2 S$ (where $S:=\left\{x \in c_{0}:\right.$ $\|x\|=1\})$. In fact we show the following stronger result.

Theorem 3. Let $G=c_{0}$ be the additive group of the classical Banach space $c_{0}$. Assume that $f: c_{0} \rightarrow \mathbb{R}$ be any (not necessarily continuous) bounded function such that

$$
\begin{cases}f(x) \leq a & \forall\|x\|=1 \\ b \leq f(x) & \forall\|x\|=2\end{cases}
$$

for some pair $a<b$ of real numbers. Then $f$ is not a tame function on the discrete copy of the group $c_{0}$.

Proof. For every $n \in \mathbb{N}$ consider the function

$$
f_{n}: c_{0} \rightarrow \mathbb{R}, x \mapsto f\left(e_{n}+x\right),
$$

where $e_{n}$ is a vector of $c_{0}$ having 1 as its $n$-th coordinate and all other coordinates are 0 . Clearly, $f_{n}=f g_{n}$ where $g_{n}=e_{n} \in c_{0}$. We have to check that $f G$ is an untame family. It is enough to show that the sequence $\left\{f_{n}\right\}_{n \in \mathbb{N}}$ in $f G$ is an independent family of functions on $c_{0}$. We have to show that for every finite nonempty disjoint subsets $I, J$ in $\mathbb{N}$ the intersection

$$
\bigcap_{n \in I} f_{n}^{-1}(-\infty, a] \cap \bigcap_{n \in J} f_{n}^{-1}[b, \infty)
$$

is nonempty.

Define $v=\left(v_{k}\right)_{k \in \mathbb{N}} \in c_{0}$ as follows: $v_{j}=1$ for every $j \in J$ and $v_{k}=0$ for every $k \notin J$. Then

(1) $v \in c_{0}$ and $\|v\|=1$.

(2) $\quad\left\|e_{i}+v\right\|=1, f_{i}(v)=f\left(e_{i}+v\right) \leq a$ for every $i \in I$.

(3) $\left\|e_{j}+v\right\|=2, f_{j}(v)=f\left(e_{j}+v\right) \geq b$ for every $j \in J$.

So we found $v$ such that

$$
v \in \bigcap_{n \in I} f_{n}^{-1}(-\infty, a] \cap \bigcap_{n \in J} f_{n}^{-1}[b, \infty)
$$

Corollary 1. The bounded RUC function

$$
f: c_{0} \rightarrow[-1,1], x \mapsto \frac{\|x\|}{1+\|x\|}
$$

is not tame on $c_{0}$ (even on the discrete copy of the group $c_{0}$ ).

Proof. Observe that $f(S)=\frac{1}{2}, f(2 S)=\frac{2}{3}$ and apply Theorem 3 .

Theorem 3 remains true for the spheres $r S$ and $2 r S$ for every $r>0$. In the case of Polish $c_{0}$ it is unclear if the same is true for any pair of different spheres around the zero. If, yes then this will imply that Tame $\left(c_{0}\right)$ does not separate the zero and closed subsets. The following question remains open even for any topological group [5,7].

Question 1. Is it true that Tame $\left(c_{0}\right)$ separates the points and closed subsets? Is it true that Polish group $c_{0}$ is Rosenthal representable? 


\section{Gromov's Compactification Need Not Be a Semigroup Compactification}

Studying topological groups $G$ and their dynamics we need to deal with various natural closed unital $G$-subalgebras $\mathcal{A}$ of the algebra $\operatorname{RUC}(G)$. Such subalgebras lead to $G$-compactifications of $G$ (so-called G-ambits, [11]). That is we have compact $G$-spaces $K$ with a dense orbit $G z \subset K$ such that the Gelfand algebra which corresponds to the compactification $G \rightarrow K, g \mapsto g z$ is just $\mathcal{A}$. Frequently but not always such compactifications are the so-called semigroup compactifications, which are very useful in topological dynamics and analysis. Compactifications of topological groups already is a fruitful research line. See among others [12-14] and references there. In our opinion semigroup compactifications deserve even much more attention and systematic study in the context of general topological group theory.

A semigroup compactification of $G$ is a pair $(\alpha, K)$ such that $K$ is a compact right topological semigroup (all right translations are continuous), and $\alpha$ is a continuous semigroup homomorphism from $G$ into $K$, where $\alpha(G)$ is dense in $K$ and the left translation $K \rightarrow K, x \mapsto \alpha(g) x$ is continuous for every $g \in G$.

One of the most useful references about semigroup compactifications is a book of Berglund, Junghenn and Milnes [9]. For some new directions (regarding topological groups) see also $[3,4,15,16]$.

Question 2. Which natural compactifications of topological groups $G$ are semigroup compactifications? Equivalently which Banach unital G-subalgebras of RUC $(G)$ are left m-introverted (in the sense of [9])?

Recall that left m-introversion of a subalgebra $\mathcal{A}$ of $\operatorname{RUC}(G)$ means that for every $v \in \mathcal{A}$ and every $\psi \in M M(\mathcal{A})$ the matrix coefficient $m(v, \psi)$ belongs to $\mathcal{A}$, where

$$
m(v, \psi): G \rightarrow \mathbb{R}, g \mapsto \psi\left(g^{-1} v\right)
$$

and $M M(\mathcal{A}) \subset \mathcal{A}^{*}$ denotes the spectrum (Gelfand space) of $\mathcal{A}$.

It is not always easy to verify left $\mathrm{m}$-introversion directly. Many natural $G$-compactifications of $G$ are semigroup compactifications. For example, it is true for the compactifications defined by the algebras $\operatorname{RUC}(G), \operatorname{Tame}(G), \operatorname{Asp}(G), \operatorname{WAP}(G)$. Of course, the 1-point compactification is a semitopological semigroup compactification for any locally compact group $G$.

As to the counterexamples. As it was proved in [3], the subalgebra $\operatorname{UC}(G):=\operatorname{RUC}(G) \cap \operatorname{LUC}(G)$ of all uniformly continuous functions is not left m-introverted for $G:=H(C)$, the Polish group of homeomorphisms of the Cantor set.

In this section we show that the Gromov's compactification of a metrizable topological group $G$ need not be a semigroup compactification.

Let $\rho$ be a bounded metric on a set $X$. Then the Gromov's compactification of the metric space $(X, \rho)$ is a compactification $\gamma: X \rightarrow P$ induced by the algebra $\mathcal{A}$ which is generated by the bounded set of functions

$$
\left\{\rho_{z}: X \rightarrow \mathbb{R}, \rho_{z}(x)=\rho(z, x)\right\}_{z \in X} .
$$

Then $\gamma$ always is a topological embedding. If $X$ is separable then $P$ is metrizable. Moreover, if $(X, \rho)$ admits a continuous $\rho$-invariant action of a topological group $G$ then $\gamma$ is a $G$-compactification of $X$; see [17].

Here we examine the following particular case. Let $G$ be a metrizable topological group. Choose any left invariant metric $d$ on $G$. Denote by $\gamma: G \rightarrow P$ the Gromov's compactification of the bounded metric space $(G, \rho)$, where $\rho=\frac{d}{1+d}$.

Consider the following natural bounded RUC function

$$
f: G \rightarrow \mathbb{R}, x \mapsto \frac{\|x\|}{1+\|x\|}
$$


where $\|x\|:=d(e, x)$. By $\mathcal{A}_{f}$ we denote the smallest closed unital $G$-subalgebra of $\operatorname{RUC}(G)$ which contains $f G=\{f g: g \in G\}$. Then $\mathcal{A}_{f}$ is the algebra which corresponds to the compactification $\gamma$. Indeed, $\rho_{g^{-1}}(x)=\rho\left(g^{-1}, x\right)=(f g)(x)$ for every $g, x \in G$.

Proof of Theorem 2

We have to prove Theorem 2.

Proof. By the discussion above, the unital $G$-subalgebra $\mathcal{A}_{f}$ of $\operatorname{RUC}(G)$ associated with $\gamma$ is generated by the orbit $f G$, where $f: G \rightarrow \mathbb{R}, f(x)=\frac{\|x\|}{1+\|x\|}$. Since $c_{0}$ is separable the algebra $\mathcal{A}_{f}$ is separable. Hence, $P$ is metrizable. If we assume that $\gamma$ is a semigroup compactification then the separability of $\mathcal{A}_{f}$ guarantees by [4] (Prop. 6.13) that $\mathcal{A}_{f} \subset \operatorname{Asp}(G)$. On the other hand, since $\operatorname{Asp}(G) \subset \operatorname{Tame}(G)$, and $f \in \mathcal{A}_{f}$ we have $f \in \operatorname{Tame}(G)$. Now observe that $f$ separates the spheres $S$ and $2 S$ and we get a contradiction to Corollary 1.

Question 3. Is it true that the Polish group $c_{0}$ admits a semigroup compactification $\alpha: c_{0} \hookrightarrow P$ such that $P$ is metrizable and $\alpha$ is an embedding? What if $P$ is first countable?

This question is closely related to the setting of this work. Indeed, by [4] (Prop. 6.13) (resp., by [4] (Cor. 6.20)) the metrizability (first countability) of $P$ guarantees that the corresponding algebra is a subset of $\operatorname{Asp}(G)$ (resp. of Tame $(G))$.

Funding: This research received no external funding.

Conflicts of Interest: The author declares no conflicts of interest.

\section{References}

1. Megrelishvili, M. Every semitopological semigroup compactification of the group $H_{+}[0,1]$ is trivial. Semigroup Forum 2001, 63, 357-370. [CrossRef]

2. W. Ruppert, Compact Semitopological Semigroups: An Intrinsic Theory; Lecture Notes in Mathematics, 1079; Springer: New York, NY, USA, 1984.

3. Glasner, E.; Megrelishvili, M. New algebras of functions on topological groups arising from G-spaces. Fundamenta Math. 2008, 201, 1-51. [CrossRef]

4. Glasner, E.; Megrelishvili, M. Banach representations and affine compactifications of dynamical systems. In Fields Institute Proceedings Dedicated to the 2010 Thematic Program on Asymptotic Geometric Analysis; Ludwig, M., Milman, V.D., Pestov, V., Tomczak-Jaegermann, N., Eds.; Springer: New York, NY, USA, 2013.

5. Glasner, E.; Megrelishvili, M. Representations of dynamical systems on Banach spaces. In Recent Progress in General Topology III; Hart, K.P., van Mill, J., Simon, P., Eds.; Atlantis Press: Amsterdam, The Netherlands, 2014; pp. 399-470.

6. Glasner, E.; Megrelishvili, M. Representations of dynamical systems on Banach spaces not containing $l_{1}$. Trans. Am. Math. Soc. 2012, 364, 6395-6424. [CrossRef]

7. Glasner, E.; Megrelishvili, M. More on tame dynamical systems. In Lecture Notes S. vol. 2013, Ergodic Theory and Dynamical Systems in Their Interactions with Arithmetics and Combinatorics; Ferenczi, S., Kulaga-Przymus, J., Lemanczyk, M., Eds.; Springer: New York, NY, USA, 2018.

8. Ferri, S.; Galindo, J. Embedding a topological group into its WAP-compactification. Studia Math. 2009, 193, 99-108. [CrossRef]

9. Berglund, J.F.; Junghenn, H.D.; Milnes, P. Analysis on Semigroups; Wiley: New York, NY, USA, 1989.

10. Rosenthal, H.P. A characterization of Banach spaces containing $\ell_{1}$. Proc. Natl. Acad. Sci. USA 1974, 71, 2411-2413. [CrossRef] [PubMed]

11. de Vries, J. Elements of Topological Dynamics; Kluwer Academic Publishers: Norwell, MA, USA, 1993.

12. Uspenskij, V.V. Compactifications of topological groups. In Proceedings of the Ninth Prague Topological Symposium, Prague, Czech Republic, 19-25 August 2001; Simon, P., Ed.; Topology Atlas: Toronto, ON, Canada, April 2002; pp. 331-346. 
13. Pestov, V. Topological groups: Where to from here? Topol. Proc. 1999, 24, 421-502.

14. Pestov, V. Dynamics of Infinite-Dimensional Groups. The Ramsey-Dvoretzky-Milman Phenomenon; University Lecture Series, 40; American Mathematical Society: Providence, RI, USA, 2006.

15. Galindo, J. On Group and Semigroup Compactifications of Topological Groups. Preprint 2010.

16. Megrelishvili, M. Fragmentability and representations of flows. Topol. Proc. 2003, 27, 497-544.

17. Megrelishvili, M. Topological transformation groups: Selected topics. In Open Problems in Topology II; Pearl, E., Ed.; Elsevier: Amsterdam, The Netherlands, 2007; pp. 423-438.

(C) 2018 by the author. Licensee MDPI, Basel, Switzerland. This article is an open access article distributed under the terms and conditions of the Creative Commons Attribution (CC BY) license (http://creativecommons.org/licenses/by/4.0/). 